\title{
Apoio psicossocial em tempos de COVID-19: experiências de novas estratégias de gestão e ajuda mútua no sul da Bahia, Brasil
}

\author{
Náira Menezes Luz Vasconcelos Cruz, Edilza Bastos de Souza, Catilene Souza Florencio \\ Sampaio, Adriana Jesus Matias dos Santos, Sarita Virolli Chaves, Ronoel Nunes da Hora, \\ Rozemere Cardoso de Souza, Josenaide Engracia dos Santos
}

\section{RESUMO}

Este artigo relata experiências de profissionais e usuários de serviços da Rede de Atenção Psicossocial (RAPS), nos municípios de Ilhéus e Itabuna, os quais estão entre os que lideram as estatísticas em número de casos da COVID-19 no estado da Bahia, Brasil. Descreve-se a criação de um grupo de WhatsApp para usuários de um Centro de Atenção Psicossocial (CAPS), com impactos na proximidade das interações entre eles, e entre eles e a equipe e a comunidade. A experiência é encorajadora, na medida em que a mútua ajuda nasce e produz autonomia e protagonismo. A outra experiência baseia-se em movimentos de uma gestora da RAPS, para articular usuários do CAPS e Atenção Primária à Saúde, para apoio emocional e respostas às demandas em momentos de pandemia e isolamento social. Nesses cenários, as experiências têm criado e fortalecido novas estratégias de saúde mental, ampliando atores sociais, ferramentas e setores, na produção do cuidado na comunidade.

Palavras-chave: Sistemas de Apoio Psicossocial; COVID-19; Saúde Mental; Apoio Socii Atenção Primária à Saúde.

\section{ABSTRACT}

This article reports experiences of professionals and service users of the Psychosocial Care Network (RAPS), in the municipalities of Ilhéus and Itabuna, which are among those that lead the statistics in the number of cases of COVID-19 in the state of Bahia, Brazil. The creation of a WhatsApp group for users of a Psychosocial Care Center is described, with impacts on the proximity and improvement of interactions between them, and between them and the team and the community. The experience is encouraging, as mutual aid is born and produces autonomy and protagonism. The other experience is based on the movements of a RAPS manager, to articulate users of CAPS and Primary Health Care, for emotional support and responses to demands in times of pandemic and social isolation. In these settings, experiences have created and strengthened new mental health strategies, expanding social actors, tools and sectors, in the production of care in the community.

Keywords: Psychosocial Support Systems; COVID-19; Mental Health; Social Support; Primary Health Care.
Revista da Rede APS 2020

Publicada em: 09/06/2020

DOI:10.14295/aps.v2i2.94

Náira Menezes L. V. Cruz (Coordenação de Saúde Mental da Prefeitura Municipal de Itabuna, Itabuna, BA, Brasil)

Edilza Bastos de Souza Catilene Souza F. Sampaio (Centro de Atenção Psicossocial - CAPS II da Prefeitura Municipal de Ilhéus, Ilhéus, BA, Brasil)

Adriana Jesus M. dos Santos Sarita Virolli Chaves Ronoel Nunes da Hora (Usuários do CAPS II da Prefeitura Municipal de Ilhéus, Ilhéus, BA, Brasil)

Rozemere Cardoso de Souza (Universidade Estadual de Santa Cruz, Ilhéus, BA, Brasil)

Josenaide Engracia dos Santos (Universidade de Brasília, Brasília, DF, Brasil)

Correspondência para: Rozemere Cardoso de Souza rcsouza@uesc.br 


\section{INTRODUÇÃO}

A pandemia de COVID-19 foi considerada uma ameaça à saúde pública global (OPAS/ OMS, 2020; ARSHAD ALI et al., 2020), e requer medidas de tratamento e de prevenção, com implicações nas dimensões física, psicológica, social, ambiental e econômica da vida humana

O isolamento social é uma medida necessária para evitar a rápida propagação da doença, porém produz, dentre outros, efeitos positivos (atitudes individuais em prol do grupo, solidárias e altruístas) e também negativos (desconfianças, atitudes preconceituosas, medo, solidão) à saúde mental da população, variando suas dimensões e implicações sociais, conforme especificidades territoriais e/ ou socioculturais. No Brasil, por exemplo, um país de alta mobilidade relacional, as medidas para não tocar, abraçar, reunir, ou seja, manter o distanciamento físico entre as pessoas pode ser considerado também distanciamento afetivo, um "dilema social" e fator desencadeante de sofrimento (NOAL, 2020; SAYURI, 2020).

Para essa situação, como tem sido a epidemia de COVID-19, considera-se que a população do país sofre um impacto psicossocial em diferentes níveis de intensidade e gravidade. A maior parte das reações é esperada, mas o agravamento e/ou duração de sentimento de insegurança e falta de controle da situação, medo, confusão, letargia, agitação e solidão, dentre outros, podem levar ao adoecimento (SCHMIDT, 2020; BRASIL, 2020).

Pessoas com transtornos mentais anteriores à pandemia estão entre os grupos vulneráveis para o agravamento de sintomas. Além destes, estima-se um aumento da incidência desses transtornos, entre um terço e metade da população, "de acordo com a magnitude do evento, o grau de vulnerabilidade psicossocial, o tempo e a qualidade das ações psicossociais na primeira fase da resposta à epidemia" (BRASIL, 2020, p. 1).
A organização de estratégias psicossociais é emergente, diante da atual pandemia, evento que, sem dúvida, representa um evento sem precedente no mundo, atingindo a todos, com casos emergindo a cada momento e desafiando os serviços de saúde. Um dos serviços que tem sido desafiado continuamente são os Centros de Atenção Psicossocial (CAPS), que oferecem atendimento às pessoas em sofrimento mental, articulado com vários serviços por meio da Rede de Atenção Psicossocial (RAPS), instituída pela Portaria no 3.088, de 23 de dezembro de 2011. Uma dessas articulações é a do CAPS com a Atenção Primária à Saúde (APS), para manejo de situações relacionadas aos pacientes com transtorno mental e à pandemia da COVID-19.

Nesse cenário, onde é comum a convivência com uma gama de estressores psicossociais, como ameaças à saúde para si e para os entes queridos, interrupções nas rotinas, separação da família, dificuldades no acesso aos medicamentos, perda de salário, isolamento social devido a programas de quarentena (TAYLOR, 2019), surgem movimentos de ações intersetoriais, de solidariedade, de cidadania e de respeito e preservação da vida, desafios centrais da RAPS.

Nessa perspectiva, este estudo descreve experiências de apoio psicossocial nos contextos de dois municípios do sul da Bahia, durante a pandemia de COVID-19, desenvolvidas por profissionais e usuários de serviços de saúde mental, numa tentativa de compartilhar e empoderar iniciativas de cuidado na comunidade, ética e responsabilidade social.

\section{RELATO DAS EXPERIÊNCIAS}

As intervenções foram conduzidas por usuários e profissionais do CAPS II, e gestora de uma RAPS, nos respectivos municípios de llhéus e Itabuna, os quais estão entre os cinco com maior coeficiente de incidência da COVID-19 por 1.000.000 de habitantes, no estado da Bahia, Brasil (BAHIA, 2020). 


\section{DesenVOLVIMENTO dO GRUPO DE MÚTUA AJUDA: O CASO DE ILHÉUS}

Em Ilhéus, a experiência foi baseada na formação de grupo online e de atendimento remoto individualizado, utilizando-se ferramentas de WhatsApp e telefone. Os administradores do grupo são três usuários, uma psicóloga e uma enfermeira.

O grupo no WhatsApp, nomeado de "Amigos do CAPS", foi uma iniciativa prévia de usuários, os quais, durante a pandemia, incluíram em sua composição dois profissionais do serviço, uma psicóloga e uma enfermeira. Hoje, o grupo conta com 32 participantes. A premissa da formação do grupo era comunicação e informação sobre as atividades do serviço e necessidades de seus integrantes. Em tempos de COVID-19, com efeitos psicossociais para todos, em especial, para pessoas com transtornos mentais, o grupo é um meio para minimizar a solidão.

Atualmente, o grupo busca também manter todos os usuários do CAPS protegidos contra o estresse crônico e problemas de saúde mental durante a pandemia. Assim, as informações e diálogos produzidos colaboram para melhor capacidade do serviço para gerenciar as situações decorrentes da pandemia.

A presença da psicóloga e enfermeira contribui para comunicação de boa qualidade e atualizações precisas de informações. Embora o CAPS II do referido município mantenha as atividades na semana, exclusivamente para consultas médicas e de Enfermagem individualizadas e acesso às medicações psicotrópicas, ressalta-se que, ao serem informadas, no Grupo, sobre qualquer situação e/ ou comportamento que indique necessidade de intervenção, esses são feitos de modo privado e individualmente, por meio do trabalho remoto.

Constata-se o grupo como um sistema de mútua ajuda para fornecer suporte, monitorar o estresse e reforçar os procedimentos de segurança, apoio social entre os usuários. 0 grupo possibilita benefícios que vão além de uma oportunidade de reunir um número plural de pessoas. Nesse sentido, estamos considerando o potencial dos grupos terapêuticos de reativar ações e percepções organizadas por formações psíquicas de propriedades grupais que não seriam compreendidas fora do acontecer grupal (KÄ̈S, 2005).

A participação da comunidade no grupo, surpreendentemente, superou todas as expectativas, pois o engajamento dos usuários foi expressivo. Vários estudos têm demonstrado que intervenções desenvolvidas em grupo, muitas vezes informais, nos quais é dada oportunidade aos membros para partilharem experiências e conhecimentos, tirar dúvidas em momentos, são úteis no alívio da sobrecarga e na diminuição dos sintomas depressivos, bem como no favorecimento de comportamentos de saúde e de procura de apoio social, os quais colaboram para melhor qualidade de vida e satisfação com a tarefa de cuidar (CHIEN et al., 2011).

Desse modo, o Grupo surge como um espaço de fortalecimento das relações e de apoio, reduzindo sentimento de solidão e fomentando a identificação e o manejo precoces das situações e/ ou comportamentos que possam sugerir nova crise durante a pandemia.

\section{TECENDO REDES DE CUIDADO EM SAÚDE}

\section{MENTAL EM TEMPOS DE PANDEMIA: A GESTÃO EM ITABUNA}

Neste tópico, são descritos os movimentos da gestão de Saúde Mental em Itabuna, a fim de alinhar o cuidado em saúde mental às medidas de prevenção e controle da COVID-19, como a necessidade primordial de distanciamento social. Diante da emergência dessa medida, refletiu-se acerca dos diversos conflitos familiares, até mesmo vínculos familiares e 
sociais fragilizados e rompidos, impossibilitando ou dificultando essa permanência em domicílio, o que se agrava quando se trata de pessoas com transtornos mentais graves e persistentes ou em uso/abuso de substâncias psicoativas. Desse modo e de imediato, foram elaboradas estratégias de enfrentamento, a fim preservar o mínimo de continuidade da assistência às pessoas com transtornos mentais atendidas pelo município, reduzindo possíveis danos à saúde desses usuários e seus familiares.

Os serviços de saúde mental disponíveis em nossa rede municipal sob a responsabilidade da Coordenação de Saúde Mental correspondem ao Ambulatório Psicossocial, aos Centros de Atenção Psicossocial, CAPS II - para pessoas com transtornos mentais graves e/ ou persistentes, 0 CAPS AD - para usuários de Álcool e outras Drogas e CAPSi - para o público infanto-juvenil, e Serviço de Demanda Jurídica. Inicialmente, atividades grupais foram suspensas nesses serviços, mantendo somente a renovação das receitas médicas, atendimento técnico restrito ao manejo das situações de agudização dos sintomas e orientações, preservando o distanciamento recomendado.

A paralisação do transporte coletivo municipal impulsionou a articulação com a Atenção Primária à Saúde (APS), com intuito de garantir a continuidade do tratamento medicamentoso e a assistência mínima às pessoas com transtornos mentais. Desse modo, ficou acertado que os médicos da APS renovariam as receitas médicas, e, em caso de dúvidas ou se a pessoa não tivesse a comprovação da receita anterior, era orientado contato com a unidade de origem, para que essa pessoa fosse atendida, para emissão de um relatório breve, contendo quadro clínico e tratamento medicamentoso, a ser enviado por e-mail. Para esclarecimentos e orientações efetivas aos profissionais da APS, foram atualizados os contatos de todas as unidades de saúde mental, endereço e e-mail.

Preocupados em garantir 0 acesso às medicações psicotrópicas, as unidades de saúde mental iniciaram uma busca ativa telefonada dos usuários, seguindo a ordem dos agendamentos das consultas médicas, o controle de enfermagem dos usuários em uso de medicação injetável e a relação dos pacientes considerados com maior possibilidade de instabilidade. Essa ação teve como objetivos: verificar os reflexos do distanciamento social e da descontinuidade das atividades terapêuticas, identificar e orientar possíveis conflitos familiares e sociais, orientar sobre a transmissão e cuidados de prevenção da COVID-19, orientar e certificar sobre a continuidade do tratamento medicamentoso, como também esclarecer dúvidas e fornecer encaminhamentos, se necessário. As psicólogas das unidades iniciaram suporte psicológico remoto, priorizando os usuários considerados de maior vulnerabilidade à desordem psíquica, dentre outras razões, por maior conflito familiar, adesão inadequada ao tratamento medicamentoso, baixo suporte familiar/social, uso/abuso de álcool e outras substâncias, práticas de automutilação, depressão grave e comportamento suicida.

$\mathrm{Na}$ busca ativa telefonada, os servidores se depararam com alguns impeditivos, dentre eles, relatos dos usuários em não conseguir atendimento nas unidades da Estratégia de Saúde da Família (ESF), devido ausência de profissional médico, afastado por adoecimento, dificuldades na comunicação entre o usuário e a unidade de saúde, resistência de alguns usuários ao acesso às unidades de saúde, deslocando-se de longa distância ao CAPS, e o mais evidente, o contato telefônico desatualizado, uma realidade presente em nossa sociedade devido à alta rotatividade do contato telefônico dos usuários, sem a devida atualização pelos serviços.

Em contrapartida, foi percebida a aproximação de usuários dos serviços de saúde mental aos serviços da atenção primária à saúde, os quais nunca ou pouco haviam sido utilizados por eles antes da pandemia. Ressalta-se a necessidade dessa aproximação, uma vez que esses usuários também possuem outras necessidades de saúde e que precisam ser assistidos em sua integralidade. Essa aproximação oportuniza o desenvolvimento de vínculos, melhorando a 
comunicação entre o profissional e usuário, contribui na construção de confiança, apresenta-se como um estímulo ao autocuidado e corresponsabilização, e um suporte ao usuário para intermediar as relações intrafamiliares e sociais.

Outra estratégia de gestão implementada foi a articulação com o Centro de Referência Especializado para População em Situação de Rua (Centro POP). Trata-se de uma instalação pública, vinculada à Secretaria de Assistência Social, que foi estruturada para prestar auxílio à população jovem e adulta que vivem em situação de rua. Parte do público é usuário dos serviços de saúde mental. Dessa forma, foi mantido contato com a coordenação local, a fim de que realizassem levantamento dos atendidos que necessitavam de receitas médicas atualizadas, conseguindo assim dar continuidade no tratamento medicamentoso. Essa ação contribuiu ao estreitamento das relações entre os serviços do Centro de Referência Especializado de Assistência Social (CREAS), Centros de Referência de Assistência Social (CRAS), Centro POP e saúde mental e ocorreu com fluidez em virtude das articulações pregressas, anteriores à pandemia, com demais departamentos de assistência social, mediante o enfrentamento de ocorrências de usuários em situação de risco pessoal ou social e até mesmo violação de direitos, que necessitavam de outros serviços especializados para garantir resolutividade de seus problemas.

O enfrentamento à COVID-19 continua e novos desafios aparecem, quando a prefeitura municipal, considerando os servidores com idade igual ao superior a 65 anos, emite um decreto proporcionando-lhes férias. 0 quadro de psiquiatras dos serviços de saúde mental contava, em sua maioria, com profissionais dentro dessa faixa etária. Desse modo, foi solicitado à direção do departamento de média e alta complexidade a contratação de um psiquiatra para realização de atendimento à demanda que surgiu durante a pandemia.
Por conseguinte, com a contratação do psiquiatra, foi orientado à APS que as situações eletivas, caracterizadas por ansiedade leve, distúrbios de relacionamento interpessoal, renovação de receitas seria realizado manejo pela ESF; nas situações de emergência psiquiátrica, caracterizadas por distúrbio do pensamento, sentimentos ou ações que envolvem risco de vida ou risco social grave, necessitando de intervenções imediatas, o manejo seria realizado pelo Serviço de Atendimento Móvel de Urgência (SAMU) e nas situações de urgência psiquiátrica, que necessitem de intervenções a curto prazo, o manejo seria realizado pelo ambulatório psicossocial. Com essas ações, foi possível realizar atendimentos de primeira consulta.

Em de abril de 2020, a Prefeitura de Itabuna, através da Secretaria Municipal de Assistência Social inaugurou o espaço Pop Acolhimento, uma casa que tem por objetivo oferecer assistência às pessoas que se encontram em situação de rua no município, oferecendo abrigamento, alimentação, higienização, garantindo proteção e direitos dos usuários. Surgiu então nova possibilidade de articulação, fornecendo atendimento e continuidade da assistência à saúde mental aos usuários em comum, bem como, orientações aos profissionais no que diz respeito ao manejo e terapia medicamentosa.

A unidade do SAMU no início da pandemia requisitou o remanejamento de um profissional psicólogo, para desenvolver estratégia de acolhimento e escuta aos seus profissionais. Essa ação possibilitou mais uma estratégia de articulação, na qual o psicólogo, após escuta, inicia um trabalho de psicoeducação, realizando encaminhamento para as unidades de saúde mental, mediante necessidade de atendimento psiquiátrico ou atendimento psicológico.

No ano de 2019, experiência de parceria da gestora com o Centro de Referência em Saúde do Trabalhador (CEREST), identificou a necessidade de avaliar condições de saúde do trabalhador, visto que em uma atividade sobre 
manejo do comportamento suicida, com Agentes comunitários de Saúde (ACS) e enfermeiros da Atenção Básica, foram relatadas diversas situações de sofrimento psíquico entre os mesmos. Diante disso, neste momento de pandemia, foi resgatada essa ação e solicitado ao CEREST o encaminhamento dos profissionais que necessitavam de atendimento, possibilitando acesso imediato à assistência no ambulatório psicossocial.

Ademais, existe também o atendimento às demandas judiciais. Nessas ocasiões, o Juiz determina a prestação de assistência psiquiátrica, internação hospitalar e/ou acompanhamento psicossocial aos indivíduos com sofrimento psíquico que requerem o serviço judicialmente. Para tanto, são necessárias visitas domiciliares com intuito de avaliar os fatores condicionantes à situação apresentada e determinar quais os encaminhamentos devidos. Sendo assim, a continuidade deste serviço foi indispensável, seguindo os protocolos de prevenção e distanciamento.

Tais experiências somam esforços em um novo grupo no WhatsApp, nomeado de Grupo de Trabalho em Saúde Mental (GTSM), criado para integrar profissionais de diferentes formações e atuação na área, coordenados por docentes da Universidade Estadual de Santa Cruz e inspirados no trabalho do Subcomitê de Saúde Mental e Apoio Psicossocial, constituído pelo Plano para Enfrentamento da COVID-19 da Universidade de Brasília (2020). Essa nova articulação tem como tendências ampliar as formas de apoio psicossocial em curso e desenvolver outras frentes de trabalho, para respostas às demandas em diferentes situações, como luto, ansiedade, depressão, apoio matricial e ações de promoção de bem estar, de populações dos municípios de entorno da universidade.

Articulando com as várias redes, as experiências aqui descritas têm como premissa a referência de Quinderé, Jorge e Franco (2014), o Modelo Rizomático, que não tem limites em si, ou seja, opera de acordo com o tensionamento dos atores que o constituem. Não é previamente estruturado e se dá em consonância com as necessidades de ajuste. Assim é a situação da pandemia aqui vivenciada. Cabe ressaltar: algumas referências para esta discussão são as novas formas de organização dos serviços de saúde mental, fruto da reestruturação do setor no Brasil.

Espera-se que este relato contribua para refletir acerca da relevância do fortalecimento da APS (NEDEL, 2020) e dos novos dispositivos de cuidado em saúde mental, assim como a retomada das Equipes de Apoio Matricial para implementação da RAPS, visando à promoção do cuidado e bem estar, nos períodos durante a pandemia e pós-pandemia.

\section{CONSIDERAÇõES FINAIS}

Considerando o exposto, neste relato de experiências, o grupo de WhatsApp é uma prática em expansão, devido a pandemia, apesar da restrição de alguns profissionais. A experiência é encorajadora, na medida em que a mútua ajuda nasce entre os usuários, fortalecendo a autonomia e protagonismo. Os poucos estudos já realizados também são encorajadores, sendo importante enfrentarmos o desafio dessa ferramenta até o momento tão pouco explorada.

A experiência da articulação do CAPS com todos os dispositivos territoriais, principalmente a APS, seguiu pontos importantes de orientação para todos, com destaque para aproximação dos usuários dos CAPS com APS, em busca de apoio emocional e a disponibilidade de medicamentos psicotrópicos genéricos essenciais, pois pessoas que vivem com problemas de saúde mental em longo prazo precisarão, ininterruptamente, de acesso ao medicamento e a interrupção repentina deve ser evitada.

O artigo ressalta a importância da inovação do cuidado através do uso das mídias sociais e teleatendimento, bem como do cenário da APS 
no cuidado em saúde mental, onde é preciso garantir o acesso ao sistema e contribuir para o fortalecimento de vínculos com as pessoas, e "entender sua situação inserida em um 'território vivo', através de sua configuração familiar e na comunidade em que vive" (NEDEL, 2020, p.14).

Assim, as experiências relatadas procuraram demonstrar que a apropriação de tecnologias, abordagens e estratégias, no cenário da COVID19, possibilitou a criação de uma rede de vínculos, fundamental para efetivação da atenção psicossocial articulada com a APS. 


\section{NOTAS E REFERÊNCIAS}

ARSHAD ALI S, BALOCH M, AHMED N, ARSHAD ALI A, IQBAL A. The outbreak of Coronavirus Disease 2019 (COVID-19)-An emerging global health threat. J Infect Public Health. 2020;13(4):644-646. doi:10.1016/j.jiph.2020.02.033. Disponível em: https://pubmed.ncbi.nlm.nih.gov/32199792/. Acesso em: 24 de abr. 2020.

BAHIA. SECRETARIA DE SAUDE DO ESTADO. Bahia tem 6.204 confirmaçoes de covid-19 e 225 óbitos Disponível em: http://www.saude.ba.gov.br/2020/05/12/bahia-tem-6-204-confirmacoes-de-covid-19-e225-obitos/. Acesso em: 29 de mai. 2020.

BRASIL, MINISTÉRIO DA SAÚDE. FUNDAÇÃO OSWALDO CRUZ. Saúde Mental e Atenção Psicossocial na Pandemia Covid-19 - Recomendações para gestores (2020). https://www.fiocruzbrasilia.fiocruz.br/wpcontent/uploads/2020/04/Sa\%C3\%BAde-Mental-e-Aten\%C3\%A7\%C3\%A3o-Psicossocial-na-PandemiaCovid-19-recomenda\%C3\%A7\%C3\%B5es-para-gestores.pdf Acesso em 27/05/2020.

CHIEN, L.Y.; CHU, H.; GUO, J.L. et al. Caregiver support groups in patients with dementia: a meta-analysis. Int J Geriatr Psychiatry, v. 26, n. 10, p. 1089-1098, 2011. [ Links ]

DELGADO, Pedro Gabriel. Reforma psiquiátrica: estratégias para resistir ao desmonte.Trab. educ. saúde, Rio de Janeiro, v. 17, n. 2, e0020241, 2019 . Available from <http://www.scielo.br/scielo.php?script=sci_arttext\&pid=S198177462019000200200\&Ing=en\&nrm=iso>. access on 27 May 2020. Epub May 06, 2019. http://dx.doi.org/10.1590/1981-7746-sol00212.

KAËS, R. La palabra y el vínculo: procesos asociativos en los grupos. Madrid: Amorrortu Editores, 2005. $357 \mathrm{p}$.

NEDEL, F. Enfrentando a COVID-19: APS forte agora mais que nunca! APS EM REVISTA, v. 2, n. 1, p. 11-16, 15 abr. 2020. Disponível em: https://apsemrevista.org/aps/article/view/68. Acesso em: 29 de mai. 2020.

NOAL, D. Recomendações Gerais, Módulo 1. In: Curso Atualização em Saúde Mental e Atenção Psicossocial na COVID-19 (Live). Acesso em: www.fiocruzbrasilia.fiocruz.br facebook.com/fiocruzbrasilia instagram.com/fiocruzbrasilia. Acesso em: 28 de mai. 2020.

ORGANIZAÇÃO PAN-AMERICANA DE SAÚDE. OMS afirma que COVID-19 é agora caracterizada como pandemia. Washington: OPAS, 11 de mar. 2020. Disponível em: https://www.paho.org/bra/index.php?option=com_content\&view=article\&id=6120:oms-afirma-quecovid-19-e-agora-caracterizada-como-pandemia\&Itemid=812. Acesso em: 24 abr. 2020.

QUINDERÉ, PHD; JORGE, MSB; FRANCO, TB. Rede de Atenção Psicossocial: qual o lugar da saúde mental?. Physis [Internet]. 2014 Mar [cited 2020 May 29]; 24(1): 253-271. Available from: http://www.scielo.br/scielo.php?script=sci_arttext\&pid=S0103-73312014000100253\&lng=en. https://doi.org/10.1590/S0103-73312014000100014

SAYURI, J. Coronavírus: qual o impacto do isolamento nas sociedades mais 'abertas' do mundo. De Toyohashi (Japão) para a BBC News Brasil, em 28 de mar. 2020. Disponível em: https://www.bbc.com/portuguese/internacional-52042839. Acesso em: 29 de mai. 2020.

SCHMIDT, B. et al. Saúde mental e intervenções psicológicas diante da pandemia do novo coronavírus (COVID-19). Estud. psicol. (Campinas), Campinas, v. 37, e200063, 2020. Available from $<$ http://www.scielo.br/scielo.php?script=sci_arttext\&pid=S0103- 
166X2020000100501\&Ing=en\&nrm=iso>. access on 28 May 2020. Epub May 18, 2020. https://doi.org/10.1590/1982-0275202037e200063.

TAYLOR, S. The Psychology ofPandemics: Preparingfor the Next Global Outbreak of Infectious Disease. Cambridge Scholars Publishing This book first published, 2019.

UNIVERSIDADE DE BRASÍLIA. Plano de Contingência em Saúde Mental e Apoio Psicossocial para Enfrentamento do Novo Coronavírus (SARS-CoV-2) para a Universidade de Brasília. Disponível em: http://www.UnB.br/images/Noticias/2020/Documentos/2020_PlanodeContigenciaSaudeMentalPsicoss ocial_COES-UnB.pdf. Acesso em: 7 junho 2020 